\title{
Investigating X-ray Emission from PPN and PN using numerical simulations
}

\author{
Matthias Stute and Raghvendra Sahai \\ Jet Propulsion Laboratory, California Institute of Technology, 4800 Oak Grove Drive, \\ Pasadena, CA 91109, USA \\ Matthias.Stute@jpl.nasa.gov Raghvendra.Sahai@jpl.nasa.gov
}

\begin{abstract}
The shaping of Pre-planetary nebulae (PPN) and Planetary Nebulae (PN) is believed to result from the interaction of a fast, collimated post-AGB wind (CFW) plowing into the slow, dense wind emitted during the AGB phase, followed by an isotropic tenuous wind during the PN phase (Sahai \& Trauger 1998). The expanding shell which forms the PPN and later the PN is expected to produce extended X-ray emission due to the large speed of the fast wind and resulting high temperatures in the shocked gas. X-ray emission was only detected in 3 of $60 \mathrm{PNs}$ observed with ROSAT, followed by a few more from CHANDRA and XMM (e.g. Guerrero et al. 2005). In the case of PPNs, there is so far only one confirmed X-ray detection (Sahai et al. 2003), although many have been observed with CHANDRA. The general problem of understanding the formation and shaping of PNs has been addressed analytically and numerically (see Balick \& Frank 2002, and references therein). X-ray emission is one of the most direct probes of the fast wind and the interaction process which drives PN formation. However, this probe has not been fully exploited in previous modeling studies. Very recently, Akashi et al. (2006, hereafter ASB06) used analytical, self-similar, spherically symmetric models to address this problem. We are performing numerical simulations with the hydrodynamics code $F L A S H$ varying the basic parameters of the fast and slow wind over an extensive parameter grid and computing the X-ray emission as a function of these parameters and the time history of the fast wind.
\end{abstract}

Keywords. planetary nebulae: general, X-rays: stars, ISM: jets and outflows, stars: mass loss, hydrodynamics

\section{Simulations \& Results}

Using Flash, we solve the differential equations of ideal hydrodynamics, and include radiative losses using the cooling functions of Sutherland \& Dopita (1993) and Dalgarno \& McCray (1972). We let a fast post-AGB wind (mass loss rate $\dot{M}_{\mathrm{f}}$, velocity $v_{\mathrm{f}}$ ) expand into a spherically symmetric, slowly expanding AGB wind $\left(\dot{M}_{\mathrm{s}}, v_{\mathrm{s}}\right)$. The values of these four parameters cover the range adopted from ASB06 for ease of comparison; we have expanded the range to fit models to observations. The expected X-ray flux is determined using the radial density and temperature profiles from the hydrodynamical simulations. The atomic database $A T O M D B$ with IDL (Smith et al. 2001) is used to calculate the emissivity. We compute the total flux in X-rays, the spectrum and emission maps.

Our 1-D spherical models are in good agreement with analytical solutions of the structure and kinematics of the hot bubble (Chevalier \& Imamura 1983, Koo \& McKee 1992ab) resulting from the wind-wind interaction. These models also support many of the analytical results of ASB06, although interesting differences occur. For example, we find that the initial phase of low X-ray luminosity, relevant for the understanding of X-rays from PPNe, is significantly shorter than in ASB06. We believe that our results are more accurate because of the self-consistent treatment of radiative cooling and hydrodynamics in our models. 

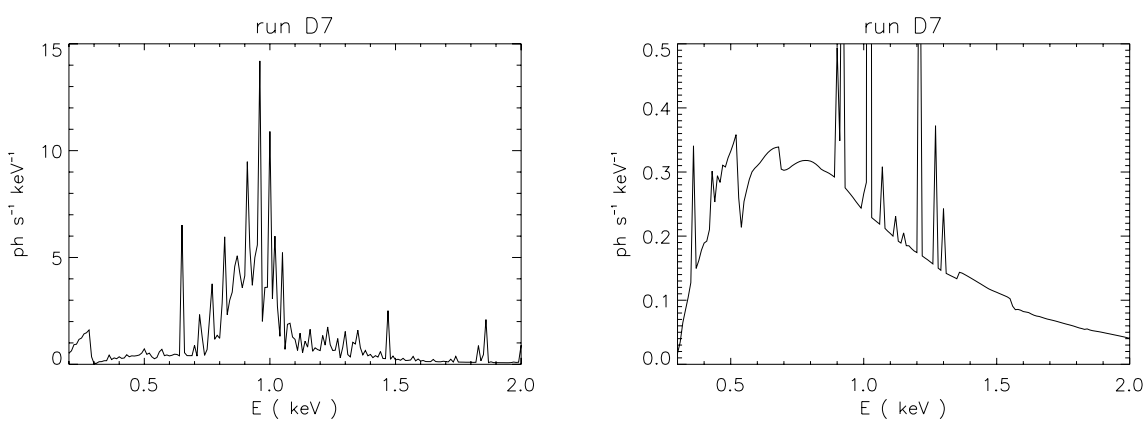

Figure 1. Theoretical spectra using model D7 at year 350 calculated with different sets of abundances (left: 14 elements with solar abundances, right: only $\mathrm{H}, \mathrm{He}, \mathrm{C}, \mathrm{Ne}$ with solar abundances)

Our 2-D spherical simulations demonstrate the importance of turbulence due to instabilities which reduces the expansion velocity and the extent of the hot bubble and therefore its total X-ray luminosity (by a factor of 1.6 relative to the 1 -D simulations).

The spherical models have been applied to the two objects BD $+30^{\circ} 3639$ and NGC40 which appear to be round. We used their observed X-ray and optical properties (the size and expansion velocity of the dense shell) to confront our models for the shaping of these PNs. We find that abundances sensitively affect the X-ray luminosity and spectrum (Fig. 1). We also find that different time histories of the fast wind may result in very similar optical and X-ray properties for a given $\mathrm{PN}$.

We are extending our simulations to collimated fast outflows, in order to model PNs detected in X-rays which show bipolar or asymmetric structures with varying degrees of collimation. The collimation of the fast wind results in the hot bubble being confined to the jet head region, significantly reducing the amount and spatial extent of the X-ray flux relative to the spherical case. Such models may thus provide an explanation for the X-ray emission from the PPN, He 3-1475, which is confined to a compact knot-like region.

\section{Acknowledgements}

The software used in this work was in part developed by the DOE-supported ASC/ Alliance Center for Astrophysical Thermonuclear Flashes at the University of Chicago. We thank V. Dwarkadas for help and discussions related to the use of the FLASH code. This work was partially funded by NASA/Chandra grants GO3-4019X and GO4-5163Z, and NASA/STScI grant HST-GO-10317.01-A.

\section{References}

Akashi, M., Soker, N., \& Behar, E. 2006, MNRAS, 368, 1706

Balick, B. \& Frank, A. 2002, ARA\&A 40, 439

Chevalier, R. A. \& Imamura, J. N. 1983, ApJ 270, 554

Dalgarno, A. \& McCray, R. A. 1972, ARA\&A 10, 375

Guerrero, M. A., Chu, Y.-H., Gruendl, R. A., \& Meixner, M. 2005, A \& A 430, L69

Koo, B. C. \& McKee, C. F. 1992, ApJ 388, 93

Koo, B. C. \& McKee, C. F. 1992, ApJ 388, 103

Sahai, R. \& Trauger, J. 1998, AJ 116, 1357

Sahai, R., Kastner, J. H., Frank, A., Morris, M., \& Blackman, E. G. 2003, ApJ 599, 87

Smith, R. K., Brickhouse, N. S., Liedahl, D. A., \& Raymond, J. C. 2001, ApJ 556, L91

Sutherland, R. S. \& Dopita, M. A. 1993, ApJS, 88, 253 